\section{Spectrophotometric techniques for the characterization of strains involved in the blue pigmentation of food: Preliminary results}

\author{
Luca Fasolato, ${ }^{1}$ \\ Nadia Andrea Andreani, ${ }^{1}$ \\ Roberta De Nardi, ${ }^{2}$ Giulia Nalotto, ${ }^{1}$ \\ Lorenzo Serva, ${ }^{2}$ Barbara Cardazzo, ${ }^{1}$ \\ Stefania Balzan, ${ }^{1}$ Lisa Carraro, ${ }^{1}$ \\ Federico Fontana, ${ }^{1}$ Enrico Novelli ${ }^{1}$ \\ ${ }^{1}$ Department of Comparative \\ Biomedicine and Food Science, and \\ ${ }^{2}$ Department of Animal Medicine, \\ Productions and Health, University of \\ Padua, Italy
}

\begin{abstract}
Near infrared spectroscopy (NIRs) and ultraviolet visible spectroscopy (UV-vis) have been investigated as rapid techniques to characterize foodborne bacteria through the analysis of the spectra of whole cells or microbial suspensions. The use of spectra collected from broth cultures could be used as a fingerprint for strain classification using a combined polyphasic approach. The aim of this study was to evaluate the feasibility of NIRs and UV-vis for the characterization of blue strains belonging to the Pseudomonas fluorescens group. The bacteria were isolated from different food matrices, including some spoiled samples (blue discoloration). Eightyone strains previously identified at the species level were grown in Minimal Bacterial Medium broth under standard conditions at $22^{\circ} \mathrm{C}$. Two biological replicates were centrifuged in order to separate the bacterial cells from the extracellular products. Six aliquots per strain were analyzed on a small ring cup in transflectance mode (680-2500 $\mathrm{nm}$, gap $2 \mathrm{~nm}$ ). A subset of 39 strains was evaluated by UV-vis to determine changes in the spectral characteristics at 48 and 72 hours. Several chemometric approaches were tested to assess the performance of NIRs and UVvis. According to the variable importance in projection (VIP), the $1892-2020 \mathrm{~nm}$ spectral region showed the highest level of discrimination between blue strains and others. Additional information was provided in the 680-886 and 1454-1768 $\mathrm{nm}$ regions (aromatic C-H bonds) and in the 2036-2134 $\mathrm{nm}$ region (fatty acids). Changes in UV-vis spectral data (at 48 and 72 hours) appear to indicate the presence of phenazine and catecholic compounds in extracellular products.
\end{abstract}

\section{Introduction}

Members of the Pseudomonas fluorescens group are involved in the deterioration of a wide variety of foods through the production of pigment, enzymes and other molecules involved in organoleptic decay (Andreani and Fasolato, 2017). This spoilage potential has been reported in a large number of well-publicized cases of several food lots being withdrawn from the market, especially cheese (e.g. blue mozzarella), resulting in economic losses and alarming consumers (Nogarol et al., 2013; RASFF, 2010). This abnormal blue discoloration has been investigated by several authors (Andreani et al., 2014; Caputo et al., 2015; Nogarol et al., 2013), but some peculiar aspects are still under debate. For instance, the chemical nature of the pigment is not well-defined, but recently matrix assisted laser desorption ionizationtime of flight mass spectrometry (MALDI-TOF MS) analysis revealed that the blue pigment produced by colored $P$. fluorescens strains is probably an indigoderivative molecule (Andreani et al., 2015). Other observations have suggested the presence of indigoidine (Caputo et al., 2015). Moreover, preliminary observations have shown that this dark blue compound is a diffusible pigment, while the final pigmented material is insoluble in water (Andreani et al., 2014, 2015).

In the present study, the feasibility of two techniques, i.e. near infrared spectroscopy (NIRs) and ultraviolet visible spectroscopy (UV-vis), was investigated in order to characterize blue strains belonging to the Pseudomonas fluorescens group. Due to their ability to produce extracellular enzymes and release a wide range of secondary bioactive metabolites, Pseudomonas fluorescens is considered an efficient study model and an effective protein manufacturing factory (Son et al., 2012). For these reasons, the use of spectra collected from broth cultures or whole cells could be used as a fingerprint for the classification of strains using a combined polyphasic approach

In recent decades, spectrophotometric techniques have been investigated as alternative and rapid tools to identify and characterize microorganisms (Smith et al. 2012). These methods usually do not require sample preparation or processing and are considered to be ecofriendly reagent-free analyses. Spectral data can be achieved from whole culture media, solid plates, purified bacterial cells, extracellular metabolites and several other matrices (Maquelin et al., 2002; Marques et al., 2015; Nakakimura et al., 2012). The collection of spectral data
Correspondence: Luca Fasolato, Department of Comparative Biomedicine and Food Science (BCA), Università degli Studi di Padova, Viale dell'Università 16, 35020 Legnaro (PD), Italy.

Tel:. +39.049.8272846 - Fax: +39.049.8272604.

E-mail: luca.fasolato@unipd.it

Key words: Pseudomonas fluorescens, Polyphasic approach, NIRs, UV-Vis spectroscopy.

Contributions: the authors contributed equally.

Conflict of interest: the authors declare no potential conflict of interest.

Funding: none.

Received for publication: 19 July 2017.

Revision received: 10 November 2017.

Accepted for publication: 11 November 2017.

This work is licensed under a Creative Commons Attribution-NonCommercial 4.0 International License (CC BY-NC 4.0).

CC Copyright L. Fasolato et al., 2018

Licensee PAGEPress, Italy

Italian Journal of Food Safety 2018; 7:6928

doi:10.4081/ijfs.2018.6928

allows for the creation of specific libraries that can be processed through multivariate analyses for the classification of foodborne pathogens, for their quantification in food matrices or for physiological studies (e.g. definition of sublethal injury cells) (Lin et al., 2004; Nakakimura et al., 2012; Smith et al., 2012). The study of the most informative wavelength regions and band patterns could increase our understanding of blue pigment production in $P$. fluorescens strains.

\section{Materials and Methods}

\section{Strains and cultural medium applied}

A total of 81 strains (field and type strains) were analyzed, which had been previously identified at the species and strain level through a multilocus sequence typing (MLST) approach (Andreani et al., 2014). Type strains and reference strains $(n=18)$ were selected from the P. fluorescens group, while the field strains were collected from different foods such as mozzarella cheese, sushi, vegetables and other ready-to-eat foods. As described by Andreani et al. (2014), a monophyletic cluster called the blue branch has been defined by MLST analysis. From this genetic cluster, nine strains producing blue pigment and four 
white strains were included in library construction.

The strains were plated on Pseudomonas Agar Base (CFC PAB; Oxoid Microbiology Products, Thermo Scientific) and incubated at $22^{\circ} \mathrm{C}$ for $24-48 \mathrm{~h}$. Pure colonies were suspended in $10 \mathrm{~mL}$ of in MBM broth $(0.7 \%$ $\mathrm{K}_{2} \mathrm{HPO}_{4}, 0.3 \% \mathrm{KH}_{2} \mathrm{PO}_{4}, 0.05 \%$ trisodium citrate, $0.01 \% \mathrm{MgSO}_{4}, 0.1 \%\left(\mathrm{NH}_{4}\right)_{2} \mathrm{SO}_{4}$, $0.2 \%$ glucose) (Boles et al., 2004) until pigment production was observed, as reported by Andreani et al. (2015). This medium was composed of salts with trisodium citrate and glucose as carbon sources and enhances the production of blue pigment. No amino acids or proteins were added, which could reduce the effects of interfering compounds present in commonly used growth media (Lin et al., 2004). According to the preliminary growth curves, the culture broths were standardized in the range of $0.8-1$ optical density (O.D.) at 600 $\mathrm{nm}$. Two biological replicates were centrifuged (3000 rpm for 30 minutes) in order to separate the bacterial cells from the extracellular products. Supernatants and pellets were processed according to the spectrophotometric technique adopted for spectral library acquisition. In the experiments conducted in the UV-vis range the supernatants and cell pellets were resuspended in physiological solution. For the NIRs analysis, only supernatants were used (Nakakimura et al., 2012).

\section{Near infrared spectroscopy}

NIRs analysis was performed using a UNITY scientific SpectraStar 2500TW laboratory spectrophotometer. Six aliquots of supernatant per strain $(200 \mu \mathrm{l})$ were analyzed on a small ring cup in transflectance mode $(680-2500 \mathrm{~nm})$ at room temperature. Reflectance data were recorded at $2 \mathrm{~nm}$ intervals and saved as $\log 1 / \mathrm{R}$ (where $\mathrm{R}$ is reflectance).

Methods based on latent variables were applied to investigate the data sets: Principal
Component Analysis (PCA) was useful as explorative system for the internal structure of the data, the samples were then categorized by Modified Partial Least Squares (MLPS). The Soft Independent Modeling of Class Analogies (SIMCA) approach was adopted to define the membership class using PCA in order to identify local models for subsequent cluster analysis (Alexandrakis et al., 2008). This approach also allowed us to predict class membership in additional samples. The first exploratory approach evaluated all wavelengths (680-2500 $\mathrm{nm}$ whole spectrum) and the potential discriminating ability for the classes of interest. The classes analyzed were: i) blue pigment producing bacteria $v s$. all non-pigmented bacteria (Model 1; Table 1), ii) blue branch members vs. other Pseudomonas species (Model Blue Branch; Table 1) and iii) between genetically related blue strains and white strains included in the blue branch (Model Blue vs. White, Table 1). Discrimination was achieved on the basis of genetic clusters to understand whether spectroscopic reading of extracellular liquids could discriminate bacteria segregated on a genetic basis. Further discrimination was performed on the basis of alteration potential, thus creating four levels depending on the ability of these strains to produce extracellular enzymes involved in lipolysis or proteolysis as well as lecithinase. Activity was rated on a scale where 0 is zero activity and 4 is maximum production (according to the data reported by Andreani et al., 2014). The accuracy of the classification was evaluated by cross-validation. The performance of each model was evaluated in terms of specificity (Sp) and sensitivity (Se). Further models were developed using the selected wavelengths based on the variable importance in projection score (VIP $>1$ ) criterion (Andersen and Bro, 2010). This index estimates the importance of each variable in the projection used in a model. A variable with a VIP score $>1$ was considered important for the model, while under this threshold the wavelengths were considered to be less informative (Model 2 and Model 3; Table 1).

\section{UV-visible spectroscopy}

UV-visible spectroscopy was applied to evaluate pigment production in a subset of strains $(n=48)$. Culture broths were examined at $48 \mathrm{~h}$ and $72 \mathrm{~h}$, according to the visible production of blue pigment in MBM. As reported by Andreani et al. (2015), the pigment was visible when the O.D. reached 0.7 at $600 \mathrm{~nm}$. Supernatants and resuspended pellets were investigated only at 72h. Measurements were performed by a Multiskan GO Microplate Reader spectrophotometer (Thermo Fisher Scientific, Waltham, Massachusetts, USA). The analysis was carried out in 96-well microtiter plates (Sarstedt, Nümbrecht, Germany) with a standardized inoculum $(200 \mu \mathrm{L})$. O.D. $600 \mathrm{~nm}$ measurements were performed on each sample and the entire spectrum was acquired (200-1000 nm with a gap of $2 \mathrm{~nm}$ ). In order to correct for possible absorbance interference from the broth, blank samples were also assessed.

Some mathematical pre-treatments were used, aiming to reduce the light scattering caused by the sample particles and to remove additional variation in the baseline shift. The standard normal variate and detrend (SNVD), smoothing, first and second derivatives were calculated using MATLAB data analysis software to determine the useful chemometric strategies. Partial least squares discriminant analysis (PLSDA) was performed using a multivariate approach in the calibration of white and blue data sets, and cross-validation was applied to test the accuracy of the model (Murray et al., 2001). The models were evaluated considering the coefficient of determination for calibration $\left(\mathrm{R}^{2}\right)$ and cross-validation $\left(\mathrm{r}^{2}\right)$, and the corresponding standard error of calibration

Table 1. SIMCA models applied for the analysis of NIRs transflectance spectra.

\begin{tabular}{|c|c|c|c|c|c|c|}
\hline Models & Pre-treatment & Range (nm) & Se & Sp & Informative wavelengths (nm) & Characteristic bands \\
\hline Model 1 & Raw & $680-2500$ & 0.61 & 0.99 & 680-956; 1004-1130; 1892-2020 & - \\
\hline Model 2 & Raw & $\begin{array}{c}680-956 \\
1004-1130 \\
1892-2020\end{array}$ & 0.61 & 0.99 & 1892-2020 & O-H stretch and deformation \\
\hline Model 3 & Raw & $1892-2020$ & 0.66 & 0.99 & $1892-1896 ; 1996-2020$ & R-NH2; C=O \\
\hline Blue Branch & Raw & $680-2500$ & 0.93 & 1 & $1996-2020$ & $\begin{array}{l}\text { O-H stretch and deformation; } \\
\text { R-NH2; C=O }\end{array}$ \\
\hline Blue vs. White & Raw & $680-2500$ & 0.89 & 0.89 & $\begin{array}{r}680-886 \\
1454-1768 \\
2036-2134\end{array}$ & $\begin{array}{l}\text { fourth overtone } \mathrm{C}-\mathrm{H} \text {; third overtone } \mathrm{C}-\mathrm{H} \text {; } \\
\text { stretch -CH3; aromatic C-H; } \\
\text { overtone O-H; aromatic C-H comb; first overtone } \\
\text {-CONH- amides; } \\
\text { CH2 } \\
\text { fatty acids }\end{array}$ \\
\hline
\end{tabular}


(SEC) and cross-validation (SECV). Moreover, Se and Sp were also calculated. As previously reported, the VIP score was adopted for the interpretation of spectral variables.

\section{Results and Discussion}

\section{Near infrared spectroscopy}

In total, 1004 raw spectra were collected from the centrifuged supernatants. The spectra did not show specific peaks according to the different Pseudomonas species analyzed or peculiar shapes related to pigment production. This observation is in agreement with the work of Nakakimura et al. (2012), who similarly did not observe noticeable differences between spectra collected from bacterial species such as Escherichia coli and Staphylococcus aureus. Some differences in the absorbance and shape of spectra were observed in the 1880$2080 \mathrm{~nm}$ range (Figure 1A). This variability was mainly related to the different biological replicates and not associated with particular strains. The two main peaks (1450 and 1940 $\mathrm{nm})$ were associated with water regions (e.g. first overtone band and $\mathrm{O}-\mathrm{H}$ stretching modes) (Dubois et al., 2005; Nakakimura et al., 2012). The peak at $2300 \mathrm{~nm}$ was linked to fatty acid moieties $\left(\mathrm{CH}_{2}\right.$ bending/stretching combinations) (Dubois et al., 2005). As a preliminary study, models were built without pre-treatment of the raw spectra (Table 1). The raw spectral information was not suitable to develop models for the classification of
Pseudomonas species or to categorize phenotypes. However, additional treatments of the spectral data allowed for classification. Only the results on the classification of blue strains are reported. To overcome multicollinearity problems, several mathematical applications were applied, and the chemometric approach reduced spectral complexity without altering the suitable information.

Model 1 investigated the possibility of classifying blue strains with respect to all the data sets. The application of the entire spectrum allowed for the classification of $87 \%$ of the blue strains. The VIP scores selected three main spectral regions (680$956 \mathrm{~nm}, 1004-1130 \mathrm{~nm}$ and 1892-2020 nm) for further analyses. Model 2 and Model 3 showed that the most informative region was the band related to the combination of $\mathrm{O}-\mathrm{H}$ stretching and deformation (1892-2020 nm). This region provided an increase in classification performance; moreover, a similar region (1996-2020 nm) was selected according to VIP in the Blue Branch model. Interestingly, the spectral fingerprinting of supernatants collected from the same genetic cluster (Blue Branch) showed features useful for a clear discrimination (Table 1 and Figure 1B). These data suggest that the composition of extracellular metabolites produced by the blue branch strains (both white and blue) are different from those produced by the other strains. The last model (Table 1, Blue vs. White) was proposed to elucidate the fine differences between supernatants obtained from the blue strains and the white strains within the blue branch. Three main spectral regions were suitable for classification purpose: $680-886 \mathrm{~nm}, 1454-1768 \mathrm{~nm}$ and 2036-2134 nm. The range from 680 to 886 $\mathrm{nm}$ includes some characteristic features, such as bands at $690-770 \mathrm{~nm}$ corresponding to the fourth overtone of $\mathrm{C}-\mathrm{H}$, those in the spectral region of $845-878 \mathrm{~nm}$ related to the third overtone of $\mathrm{C}-\mathrm{H}$ stretching (Alexandaxis et al., 2008) and the band located at $880 \mathrm{~nm}$ related to aromatic $\mathrm{C}-\mathrm{H}$ bonds. The region from 1454 to $1768 \mathrm{~nm}$ is mainly associated with the first overtone of $\mathrm{O}-\mathrm{H}(1450 \mathrm{~nm})$, but other bands overlap in this region, such as the aromatic $\mathrm{C}-\mathrm{H}$ combination band $(1446 \mathrm{~nm})$ and the first overtone of N-H (Dubois et al., 2005). The bands at around 1632 and $1756 \mathrm{~nm}$ are putatively associated with the first overtone of -CONH- found in secondary and primary amides, e.g. DNA/RNA (Marques et al., 2015). Moreover, a contribution from the fatty acid fraction was observed (1734-1765 $\mathrm{nm}$; first overtone of $\mathrm{CH}_{2}$ ). The region from 2036 to $2134 \mathrm{~nm}$ is close to the specific absorption region related to alkenes $\left(\mathrm{CH}_{2}\right.$ stretching and bending) and unsaturated fatty acids (Dubois et al., 2005; Marques et al., 2015). These data indicate that supernatants from closely related white and blue strains (ascribed to the blue branch cluster) were composed of a different array of molecules including fatty acids, aromatics and amides.

\section{UV-visible spectroscopy}

UV-visible spectroscopy was applied to study the broth cultures during the production of blue pigment. Table 2 shows the PLSDA models developed in different matrices; SNVD was the only pre-treatment able increase the calibration performance. In general, better classification (sensitivity and

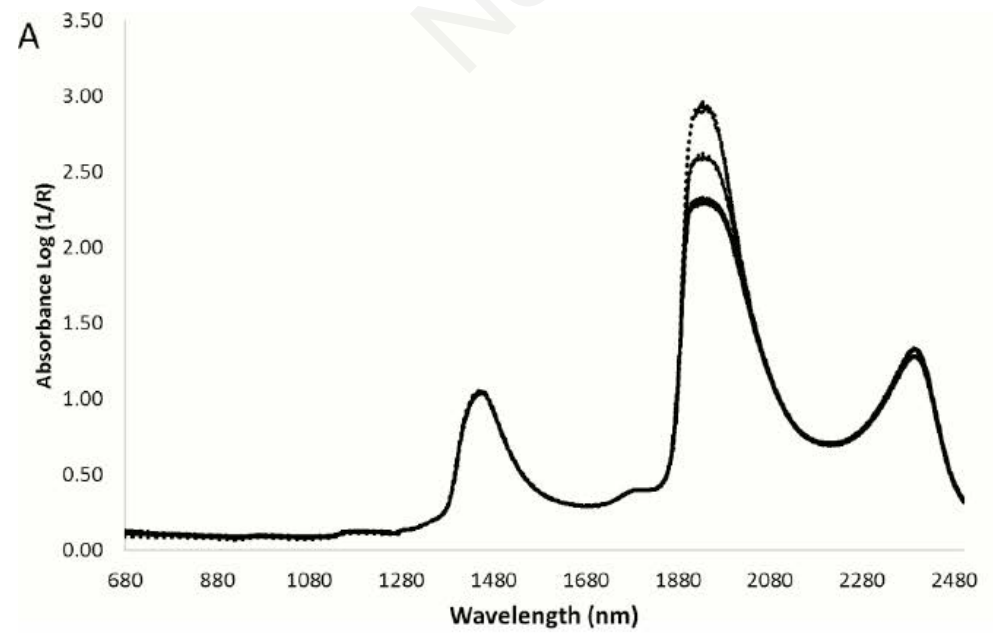

B

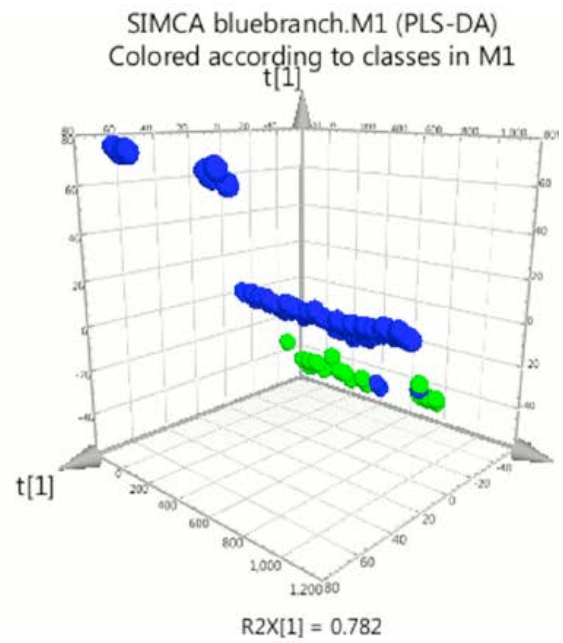

Figure 1. A) Near infrared spectra collected from blue and white strains; B) representation of the SIMCA model for blue branch raw spectral data (wavelength range 680-2480) $\mathrm{nm}$; Pseudomonas ascribed to the blue branch are indicated in green. 
specificity) was provided by spectra collected from broth cultures after $72 \mathrm{~h}$ of growth and from supernatants. These data suggest that extracellular metabolites play a key role in the differentiation between blue strains and other Pseudomonas strains.

Figure 2 shows three common spectra collected from blue strains in comparison with one spectrum from a white strain of $P$. fluorescens. Moreover, the reference spectrum of indigo $(0.1 \mathrm{mg} / \mathrm{ml}$; Sigma catalog number 229296) in MBM is also reported. Gray bands show the VIP scores for each model; only values $>1$ are shown.

The most important features selected in the broth cultures at $48 \mathrm{~h}$ ranged between 432 to $692 \mathrm{~nm}$. In this range, a specific absorption band at $600 \mathrm{~nm}$ is associated with the catechol groups of pyoverdines (Sánchez et al., 2005). Pyoverdines are the major siderophores released by Pseudomonas; these molecules are yellow-green fluorescent pigments also known as fluorescein. This family of iron chelators is formed by three different structural parts, where a quinoline chromophore group is bound to a peptide chain and to a dicarboxylic acid or a dicarboxylic amide (Tank and Saraf, 2010). The spectral information collected at $48 \mathrm{~h}$ suggested different behaviors regarding $\mathrm{Fe}^{3+}$ chelation in the broth cultures of blue strains with respect to the other Pseudomonas strains (Figure 2A).

After $72 \mathrm{~h}$, other spectral regions were involved in the classification of broth cultures (Figure 2B). The classical
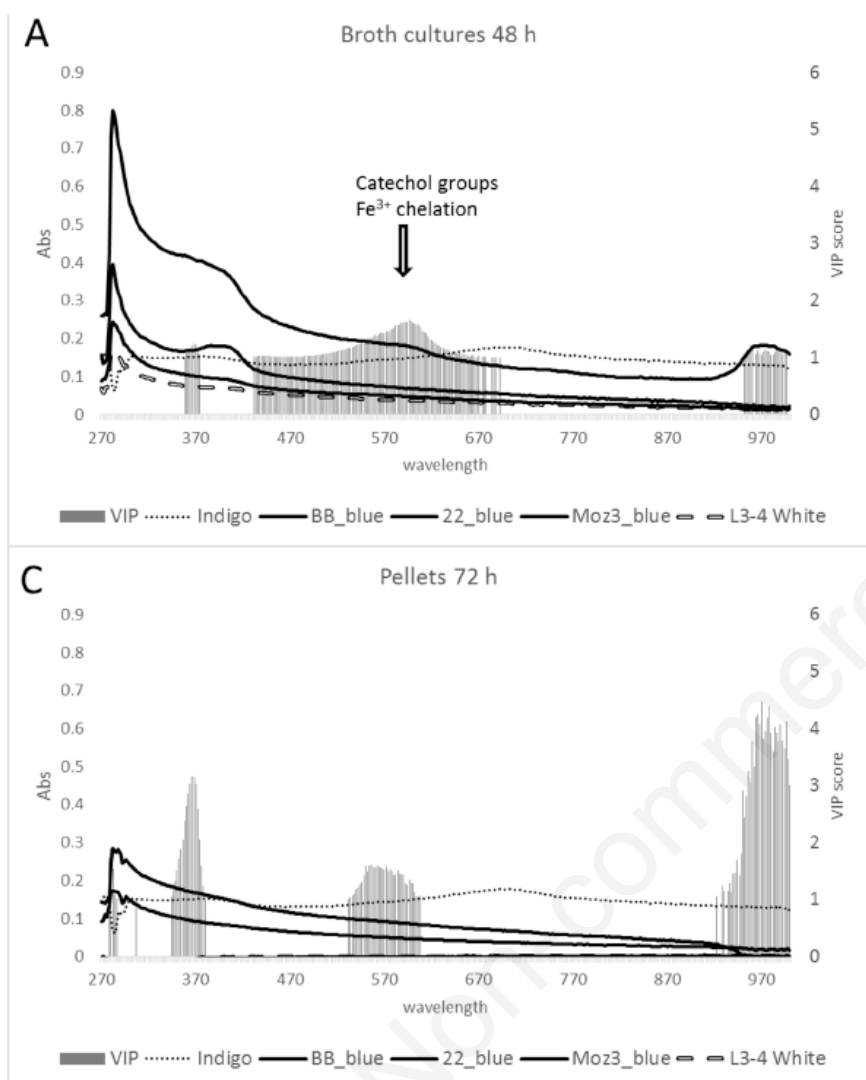

B

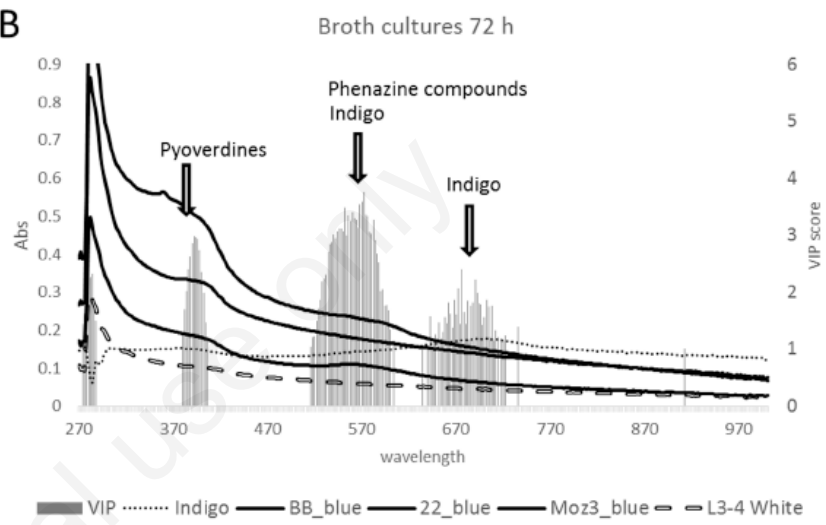

D

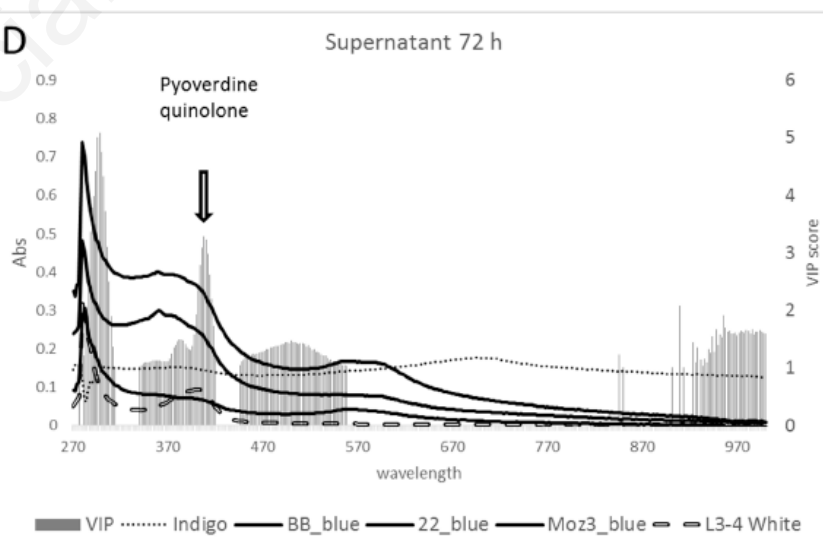

Figure 2. A) UV visible PLS models of broth cultures at $48 \mathrm{~h}$; B) broth cultures at $72 \mathrm{~h}$; C) pellets and D) supernatants. Dark lines represent the raw spectra of some blue strains and the dotted line represents the spectrum of a white strain. The absorption spectrum of commercial indigo is also shown. Gray bars indicate VIP scores $>1$.

Table 2. UV visible spectra: performance of the PLS models.

\begin{tabular}{|c|c|c|c|c|c|}
\hline Model & Pre-treatment & Se & Sp & $\mathbf{R}^{2}$ & RMSEC \\
\hline Broth cultures $48 \mathrm{~h}$ & $\begin{array}{l}\text { Raw } \\
\text { SNV }\end{array}$ & $\begin{array}{l}0.33 \\
0.78\end{array}$ & $\begin{array}{l}0.90 \\
0.73\end{array}$ & $\begin{array}{l}0.13 \\
0.21\end{array}$ & $\begin{array}{l}0.39 \\
0.37\end{array}$ \\
\hline Broth cultures $72 \mathrm{~h}$ & $\begin{array}{l}\text { Raw } \\
\text { SNV }\end{array}$ & $\begin{array}{l}0.89 \\
0.78\end{array}$ & $\begin{array}{l}0.93 \\
0.93\end{array}$ & $\begin{array}{l}0.44 \\
0.40\end{array}$ & $\begin{array}{l}0.31 \\
0.32\end{array}$ \\
\hline Supernatants $72 \mathrm{~h}$ & $\begin{array}{l}\text { Raw } \\
\text { SNV }\end{array}$ & $\begin{array}{l}0.80 \\
0.90\end{array}$ & $\begin{array}{l}0.93 \\
0.86\end{array}$ & $\begin{array}{l}0.60 \\
0.60\end{array}$ & $\begin{array}{l}0.27 \\
0.29\end{array}$ \\
\hline Pellets $72 \mathrm{~h}$ & $\begin{array}{l}\text { Raw } \\
\text { SNV }\end{array}$ & $\begin{array}{l}0.63 \\
0.38\end{array}$ & $\begin{array}{l}0.96 \\
0.93\end{array}$ & $\begin{array}{l}0.22 \\
0.35\end{array}$ & $\begin{array}{l}0.36 \\
0.35\end{array}$ \\
\hline
\end{tabular}

RMSEC: root mean squared error of calibration. Raw: raw spectrum; SNV: standard normal variate and detrend pre-treatment. $\mathrm{R}^{2}$ : coefficient of determination in calibration. Se and Sp: sensitivity and specificity. 
fingerprint of pyoverdine was related to the quinolone moiety $(390 \mathrm{~nm}$; Magro et al., 2016). The wavelengths around 570 and 670 $\mathrm{nm}$ defined the different composition of the broth cultures, probably related to pigment production. Chromophore groups of certain pigments such as violacein or phenazine compounds show absorbance peaks in the region around $580 \mathrm{~nm}$ (Gibson et al., 2009; Yuan et al., 2008); moreover, a similar UVvis spectrum of indigo produced by Pseudomonas sp. was described by Dua et al. (2014). The reference spectrum of a commercial indigo showed a peak at $696 \mathrm{~nm}$ (Figure 2). This spectral region was selected as informative by the VIP scores (Figure 2B). The peak of another cognate pigment, i.e. purified indigoidine, has been observed at $612 \mathrm{~nm}$ (Cude et al., 2012).

According to the spectrum shape, the shift from 48 to the $72 \mathrm{~h}$ showed that the blue strains released more siderophores than their white counterparts did. The specific peak and shoulder at $570-580 \mathrm{~nm}$ could be considered as a specific marker for blue pigment. However, this fingerprint was present only after $72 \mathrm{~h}$ of growth, corresponding to the stationary phase, suggesting the release of a secondary metabolite.

This information was lost in the resuspended pellets as shown in Figure 2C and as previously reported in Table 2 . The data suggest that the microbial cell spectrum was not appropriate for this study. As observed in previous studies (Andreani et al., 2014, 2015), the blue pigment is a diffusible compound that not tightly associated with cells. In the late stationary phase (at $120 \mathrm{~h}$ ), dark aggregates are removed by centrifugation. This suggests complex behavior of this compound that has been described as insoluble in water (Andreani et al., 2015).

The specific peak at $570 \mathrm{~nm}$ was maintained in the supernatant spectra collected at $72 \mathrm{~h}$ (Figure 2D). Moreover, the wavelengths corresponding to the quinolone absorption band were considered to be informative. Proteins also affect the first part of the spectrum with a specific peak at around $280 \mathrm{~nm}$. Other additional information can be correlated to molecules containing aromatic moieties such as a pyochelin-like molecule or a secondary unidentified siderophore (310 nm) (Sokol et al., 1992).

\section{Overall considerations}

These data are in agreement with the results described by Andreani and coworkers (2015) that reported an up-regulation of genes involved in iron uptake in blue strains. The bands defined by the NIRs analysis could be also associated with siderophore molecules as described by the pyoverdine structure formed by 2,3-diamino-6,7dihydroxyquinoline, a peptidic moiety and an acyl from a dicarboxylic acid or amide (Cézard et al., 2015). However, the supernatants collected from the blue branch showed common spectral features that will require a more in-depth chemometric approach to define more descriptive wavelengths. Furthermore, the presence of aromatic molecules could be also correlated with pigment production. The presence of spectral regions related to fatty acids could be due to the presence of outer membrane vesicles that show specific peaks related to lipids in Fourier transform infrared (FTIR) spectroscopy (Carlsson, 2012).

Interestingly, after $48 \mathrm{~h}$ of growth, $\mathrm{Fe}^{3+}$ chelation was more evident in blue strains than in the overall population. It is likely that phenomenon highlights the variable efficiency of pyovrerdine secreted by these strains and could reflect a difference in the structure of this iron chelator. To date, a wide number of strain-specific or species-specific pyoverdines have been described in Pseudomonas (Cézard et al., 2015), but further characterization of this siderophore is required. After 72 hours, a larger amount of pyoverdine was released by the pigmented strains. This increase in siderophores was accompanied by the presence of additional chromophore molecules. The blue pigment is probably an indigoid molecule with a specific peak at $570 \mathrm{~nm}$ and the dye is released into the broth during the late phases of growth

\section{Conclusions}

The feasibility of two different spectrophotometric techniques were investigated. NIRs and UV-visible spectra provided interesting information concerning the characterization and the production of blue pigment. Spectra are useful for strain classification regarding this peculiar spoilage activity. These libraries could be applied for further taxonomic studies; however, the dataset needs to be improved in order to increase the performance of identification. Blue strains therefore exhibit different behavior from other Pseudomonas. This phenomenon could imply a different competitiveness in the food environment. Moreover, new calibrations could be performed during challenge experiments with blue Pseudomonas to study the dynamics of pigment production in real food matrices (e.g. the governing liquid of mozzarella cheese).

\section{References}

Alexandrakis D, Downey G, Scannell AGM, 2008. Detection and identification of bacteria in an isolated system with nearinfrared spectroscopy and multivariate analysis. J Agric Food Chem 56:3431-7.

Andersen CM, Bro R, 2010. Variable selection in regression - a tutorial. J Chemom 24:728-37.

Andreani NA, Fasolato L, 2017. Chapter 2 Pseudomonas and Related Genera, In: Antonio Bevilacqua A, Corbo MR, Sinigaglia M, eds. The Microbiological Quality of Food $1^{\text {st }}$ Edition. Foodborne Spoilers. Woodhead Publishing Series in Food Science, Technology and Nutrition, Woodhead Publishing, pp 2559

Andreani NA, Carraro L, Martino ME, Fondi M, Fasolato L, Miotto G, Magro M, Vianello F, Cardazzo B, 2015. A genomic and transcriptomic approach to investigate the blue pigment phenotype in Pseudomonas fluorescens. Int $\mathrm{J}$ of Food Microbiol 213:88-98.

Andreani NA, Martino ME, Fasolato L, Carraro L, Montemurro F, Mioni R, Bordin P, Cardazzo B, 2014. Tracking the blue: a MLST approach to characterize the Pseudomonas fluorescens group. Food Microbiol 39:116-26.

Caputo L, Quintieri L, Bianchi DM, Decastelli L, Monaci L, Visconti A, Baruzzi F, 2015. Pepsin-digested bovine lactoferrin prevents Mozzarella cheese blue discoloration caused by Pseudomonas fluorescens. Food Microbiol 46:15-24.

Carlsson S, 2012. Surface characterization of gram-negative bacteria and their vesicles using fourier transform infrared spectroscopy and dynamic light scattering. PhD Dissertation.

Cézard C, Farvacques N, Sonnet P, 2015. Chemistry and biology of pyoverdines, Pseudomonas primary siderophores. Curr Med Chem 22:165-86.

Cude WN, Mooney J, Tavanaei AA, Hadden MK, Frank AM, Gulvik CA, May AL, Buchan A, 2012. Production of the antimicrobial secondary metabolite indigoidine contributes to competitive surface colonization by the marine roseobacter Phaeobacter sp. strain Y4I. Appl Environ Microb 78:4771-80.

Dua A, Chauhan K, Pathak H, 2014. Biotransformation of indigo pigment by indigenously isolated Pseudomonas sp. HAV-1 and assessment of its antioxidant property. Biotechnol Res Int 2014:1e6.

Dubois J, Lewis E, Fry F, Calvey E, 2005. Bacterial identification by near-infrared 
chemical imaging of food-specific cards. Food Microbiol 22:577-83.

Gibson J, Sood A, Hogan DA, 2009. Pseudomonas aeruginosa-Candida albicans interactions: localization and fungal toxicity of a phenazine derivative. Appl Environ Microb 75:504-13.

Lin M, Al-Holy M, Al-Qadiri H, Kang DH, Cavinato AG, Huang Y, Rasco BA, 2004. Discrimination of intact and injured Listeria monocytogenes by Fourier transform infrared spectroscopy and principal component analysis. J Agr Food Chem 52:5769-77.

Magro M, Fasolato L, Bonaiuto E, Andreani NA, Baratella D, Corraducci V, Miotto G, Cardazzo B, Vianello F, 2016. Enlightening mineral iron sensing in Pseudomonas fluorescens by Surface Active Maghemite Nanoparticles: Involvement of the OprF porin. BBA General Subjects 1860:2202-10.

Maquelin K, Kirschner C, Choo-Smith LP, van den Braak N, Ph. Endtz H, Naumann D, Puppels GJ, 2002. Review article Identification of medically relevant microorganisms by vibrational spectroscopy. J Microbiol Methods 51:255-71.

Marques AS, Moraes EP, Júnior MAA., Moura AD, Neto VFA, Neto RM, Lima
KMG, 2015. Rapid discrimination of Klebsiella pneumoniae carbapenemase 2 - producing and non-producing Klebsiella pneumoniae strains using near-infrared spectroscopy (NIRS) and multivariate analysis. Talanta 134:12631.

Murray I, Aucott LS, Pike IH, 2001. Use of discriminant analysis on Vis and near infrared reflectance spectra to detect adulteration of fishmeal with meat and bone meal. J Near Infrared Spec 9: 297311.

Nakakimura Y, Vassileva M, Stoyanchev T, Nakai K, Osawa R, Kawano J, Tsenkova R, 2012. Extracellular metabolites play a dominant role in near-infrared spectroscopic quantification of bacteria at food-safety level concentrations. Anal Methods 4:1389.

Nogarol C, Acutis PL, Bianchi DM, Maurella C, Peletto S, Gallina S, Adriano D, Zuccon F, Borrello S, Caramelli M, Decastelli L, 2013. Molecular characterization of Pseudomonas fluorescens isolates involved in the Italian "blue mozzarella" event. J Food Protect 76:500-4.

RASFF, 2010. Rapid Alert System for Food and Feed. Annual Report.

Sánchez P, Gálvez N, Colacio E, Miñones E,
Domínguez-Vera JM, 2005. Catechol releases iron (III) from ferritin by direct chelation without iron (II) production. Dalton Trans 4:811-3.

Smith JM, Huffman DE, Acosta D, Serebrennikova Y, García-Rubio L, Leparc GF, 2012. Reagent-free bacterial identification using multivariate analysis of transmission spectra J Biomed Opt 17:1070021-9.

Sokol ACJ, Lewis J, Dennis J, 1992. Isolation of a novel siderophore from Pseudomonas cepacia. J Med Microbiol 36:184-9.

Son M, Moon Y, Oh MJ, Han SB, Park KH, Kim JG, Ahn JH, 2012. Lipase and protease double-deletion mutant of Pseudomonas fluorescens suitable for extracellular protein production. Appl Environ Microb 78:8454-62.

Tank N, Saraf M, 2010. Salinity resistant plant growth promoting rhizobacteria ameliorates sodium chloride stress on tomato plants. J Plant Interact 5:51-8.

Yuan L, Liyan W, Yuan X, Chong Z, XinHui X, Kai L, Zhidong Z, Yong L, Guifeng Z, Jingxiu B, Zhiguo S, 2008. Production of violet pigment by a newly isolated psychrotrophic bacterium from a glacier in Xinijiang, China. Biochem Eng J 43:135-41. 